\title{
Probing dipole radiation from binary neutron stars with ground-based laser-interferometer and atom-interferometer gravitational-wave observatories
}

\author{
Junjie Zhao, ${ }^{1}$ Lijing Shao, ${ }^{2,3, *}$ Yong Gao, ${ }^{4,2}$ Chang Liu, ${ }^{4,2}$ Zhoujian Cao, ${ }^{5}$ and Bo-Qiang Ma ${ }^{1,6,7}$ \\ ${ }^{1}$ School of Physics and State Key Laboratory of Nuclear Physics and Technology, Peking University, Beijing 100871, China \\ ${ }^{2}$ Kavli Institute for Astronomy and Astrophysics, Peking University, Beijing 100871, China \\ ${ }^{3}$ National Astronomical Observatories, Chinese Academy of Sciences, Beijing 100012, China \\ ${ }^{4}$ Department of Astronomy, School of Physics, Peking University, Beijing 100871, China \\ ${ }^{5}$ Department of Astronomy, Beijing Normal University, Beijing 100875, China \\ ${ }^{6}$ Collaborative Innovation Center of Quantum Matter, Beijing, China \\ ${ }^{7}$ Center for High Energy Physics, Peking University, Beijing 100871, China
}

(Dated: August 19, 2021)

\begin{abstract}
Atom-interferometer gravitational-wave $(\mathrm{GW})$ observatory, as a new design of ground-based GW detector for the near future, is sensitive at a relatively low frequency for GW observations. Taking the proposed atom interferometer Zhaoshan Long-baseline Atom Interferometer Gravitation Antenna (ZAIGA), and its illustrative upgrade $(\mathrm{Z}+)$ as examples, we investigate how the atom interferometer will complement ground-based laser interferometers in testing the gravitational dipole radiation from binary neutron star (BNS) mergers. A test of such kind is important for a better understanding of the strong equivalence principle laying at the heart of Einstein's general relativity. To obtain a statistically sound result, we sample BNS systems according to their merger rate and population, from which we study the expected bounds on the parameterized dipole radiation parameter $B$. Extracting BNS parameters and the dipole radiation from the combination of ground-based laser interferometers and the atom-interferometer ZAIGA/Z+, we are entitled to obtain tighter bounds on $B$ by a few times to a few orders of magnitude, compared to ground-based laser interferometers alone, ultimately reaching the levels of $|B| \lesssim 10^{-9}$ (with ZAIGA) and $|B| \lesssim 10^{-10}$ (with Z+).
\end{abstract}

\section{INTRODUCTION}

Einstein's theory of general relativity (GR) has been tested for more than a century via a great number of accurate experiments [1]. In GR gravitation is described only by a spin2 tensor field, $g_{\mu \nu}[2]$, with the corresponding gravitational emission from compact binary coalescences dominated by the quadrupolar radiation [3, 4]. For the radiative tests in GR, the Hulse-Taylor binary pulsar offered the first empirical evidence for the existence of gravitational waves (GWs) [5]. The first direct detection at the Earth of a GW signal, GW150914, has been announced by the Laser Interferometer GW Observatory (LIGO) Scientific Collaboration and Virgo Collaboration [6]. Since then, the number of GW events has expanded significantly in the past five years [7, 8] and they have stimulated fruitful researches concerning our gravitational Universe.

Although GR passes all the existing tests with flying colors, there remain some well-motivated alternative gravity theories, including one or more extra degrees of freedom other than $g_{\mu \nu}$ in the gravitational sector. These theories have a demonstrated potential to solve problems in the inflation of the Universe, dark energy, as well as to serve as a prototype for a unified theory of quantum gravity [9]. Different from $\mathrm{GR}$, in some of these alternative theories, dipole gravitational radiation from coalescing binaries is no longer strictly forbidden. Meanwhile, there are good theoretical supports that a nonzero dipole radiation violates the strong equivalence principle (SEP), which is at the heart of GR [10-12]. Therefore, in principle, searching for a dipole radiation directly tests the

\footnotetext{
*Corresponding author: 1shao@pku.edu.cn
}

SEP [12]. As a result, some aspects of strong-field gravity closely related to the SEP can be probed from the dipole radiation tests in binary pulsars and compact binary coalescences.

In the quasi-stationary strong-field regime, binary pulsar systems are powerful testbeds for gravity, where at least one component of the binary is strongly self-gravitating and the characteristic orbital speed of the binary is small while compared with the light speed [13]. Due to the extremely precise technology, the so-called pulsar timing, the existence of dipole radiation has been excluded to high confidence in these systems [14-18].

In recent years, the advanced LIGO (AdvLIGO) and the advanced Virgo (AdV) together have discovered a bulk of GW events [7, 8], which sparked great enthusiasm in fields of gravity tests [19-25]. Those discoveries make it possible to investigate various properties of gravitation in highly-dynamical, strong-field, as well as radiative regimes $[13,26]$. GWs have become a new testbed for testing strong-field gravity, in addition to the binary pulsar systems [27]. For some alternative theories, the additional gravitational degrees of freedom not only change the orbital binding energy of binaries, but also contribute to the GW waveform significantly in such strongfield regimes [16, 28]. Therefore, GWs with the dipoleradiation imprints could be detected or constrained via GW observations.

The dipole-radiation effect, corresponding to the -1 postNewtonian (PN) contribution, enters the GW waveform at a lower order than the canonical quadrupolar radiation in GR. ${ }^{1}$ Therefore, the dipole radiation is relatively more significant

\footnotetext{
${ }^{1}$ The correction of $n \mathrm{PN}$ refers to $O\left((v / c)^{2 n}\right)$ corrections with respect to the Newtonian order, where $v$ is the characteristic relative speed in the binary.
} 
at a lower GW frequency. In principle, the GW observatories with a lower accessible frequency could obtain more GW information about negative PN corrections. Such reasoning applies to the dipole radiation in this work.

Until now, LIGO Scientific Collaboration and Virgo Collaboration (LVC) have officially announced 50 compact binary coalescences in total from observing runs $\mathrm{O} 1, \mathrm{O} 2$, and O3a [7, 8]. Recently, LVC is upgrading their detectors and planning to launch the observing run $\mathrm{O} 4$ in late 2021 or early 2022 [29]. In addition, the Kamioka GW Detector (KAGRA) [30, 31], which is built in Japan, joined O3 on October 4, 2019, and the four-detector network has officially begun [29]. Besides, more future GW detectors are proposed worldwide, including LIGO-India, ${ }^{2}$ and the third-generation GW detectors, the Cosmic Explorer (CE) led by the United States $[32,33]$ and the Einstein Telescope (ET) led by Europe [25, 34].

Over the coming decades, the ground-based detectors, AdvLIGO, AdV, and KAGRA will upgrade their instruments further, and eventually, the third-generation detectors will be online. By that time, hundreds of thousands of stellar-mass binaries can be discovered across the GW frequency band in $10-10^{3} \mathrm{~Hz}$ [33]. In the 2030s, the space-borne detectors like Laser Interferometer Space Antenna (LISA) [35], Taiji [36] and TianQin [37] will enable the GW observations of the massive black holes (BHs) in the range of GW frequency $10^{-4}$ $10^{-1} \mathrm{~Hz}$. Benefiting from larger interferometer arms and quieter space environment, the space-borne detectors are suitable to investigate the low-frequency GW sciences by design. Sesana [38] studied the prospects for multi-band GW observations with AdvLIGO and LISA. It is shown that LISA can inform AdvLIGO in advance when and where binary $\mathrm{BH}(\mathrm{BBH})$ coalescences will happen. Barausse et al. [26] used the multiband observations of massive BBHs in AdvLIGO and LISA to predict the projected bounds on dipole radiation.

Given the above detectors, there remains a frequency gap spanning $10^{-1}-10 \mathrm{~Hz}$. To fulfill it, a bulk of decihertz GW detectors are proposed, broadly speaking including (i) space-borne laser interferometer detectors like the Decihertz Observatory (DO) [39, 40] and the DECihertz laser Interferometer GW Observatory (DECIGO) [41, 42], and (ii) ground-based atom interferometer detectors. Recently, Liu et al. [43] investigated the projected constraints on dipole radiation from AdvLIGO, LISA and the spaceborne decihertz detectors. Similar tests of GR via the multi-band observations involving decihertz detectors were also investigated in Refs. [44, 45]. Concerning decihertz $\mathrm{GW}$ detectors on the ground, atom interferometers-such as Zhaoshan Long-baseline Atom Interferometer Gravitation Antenna (ZAIGA) [46], Matter wave-laser based Interferometer Gravitation Antenna (MIGA) [47], and a nextgeneration Atomic Interferometric Observatory and Network (AION) [48] — are proposed to investigate GWs in the fre-

Correspondingly, the quadrupolar radiation is $0 \mathrm{PN}$ contribution in this convention.

${ }^{2}$ https://dcc. ligo.org/LIGO-M1100296/public quency range of $0.1-1 \mathrm{~Hz}$. Recently, a creative concept for a lunar-based laser-interferometer GW detector, GW Lunar Observatory for Cosmology (GLOC) [49], is proposed with a greater sensitivity. For the first time, we will study in detail the prospects of testing dipole radiation with those groundbased decihertz GW detectors.

Multi-band observations from space-borne detectors and ground-based laser-interferometer detectors have enormous abilities to test the dipole radiation [26, 43, 44, 50]. However, actual functioning schedules of these detectors will introduce big uncertainties in performing multi-band tests, and to explore as many possibilities as possible, in this work we consider ground-based atom-interferometer GW detectors and ground-based laser-interferometer detectors to test the dipole radiation. In principle, these atom-interferometer $\mathrm{GW}$ observatories record the GW signals earlier than AdvLIGO, CE, and ET, due to their lower operating frequencies. They can be made use of to bound the dipole radiation tighter. Dipole radiation tests can come from BBH systems or binary neutron star (BNS) systems [26]. Both scenarios have unique roots in specific theories. We focus on BNS systems in our study. On one hand, in some classes of alternative theories of gravity, dipole GW emission from BBHs is usually absent because of the no-hair theorem [19,51]. On the other hand, as shown by Damour and Esposito-Farèse [52], some strong-field behaviors related to dipole radiation only happen in neutron stars, and they cannot be studied with BBHs. For some theories, probing dipole radiation from BNSs could reveal unique properties of gravitation that BBHs hardly provide. This kind of study is complementary to studies that probe the dipole radiation from BBH systems in some other classes of gravity theories where BHs can be scalarized.

In our study, we use one of the modest ground-based atominterferometer detectors, ZAIGA, as an example. As we will see, limited by the influence of Newtonian noise, ZAIGA alone hardly detects GWs from distant BNS sources. In addition, we consider an imaginary upgrade, what we call " $\mathrm{Z}+$ ", whose strain sensitivity is improved by an order of magnitude compared to that of ZAIGA (see e.g. Ref. [53]). In total, we consider AdvLIGO and its updates, CE, ET, ZAIGA, and $\mathrm{Z}+$ in our study. For statistical significance, we simulate ensembles of BNSs according to the population properties of BNSs [7]. We perform the parameter estimation to obtain the bounds on dipole radiation from these systems for different detectors. As discussed earlier, due to the lower accessible frequency of ZAIGA/Z+, the effect of $-1 \mathrm{PN}$ correction can be constrained rather well. The joint bounds show that, ZAIGA/Z+ and other ground-based laser-interferometer detectors could complement each other in testing the gravitational dipole radiation for BNS systems. Especially, the constraints on the dipole radiation from AdvLIGO and CE can be tighter with the help of ZAIGA/Z+. We consider this as an extra science motivation for atom-interferometer detectors [46].

The organization of the paper is as follows. In Sec. II, we briefly introduce GW observatories that are used in our study. Section III reviews the modified GW waveform with additional dipole radiation in the parameterized post-Einsteinian (ppE) framework. In Sec. IV, we consider the population 


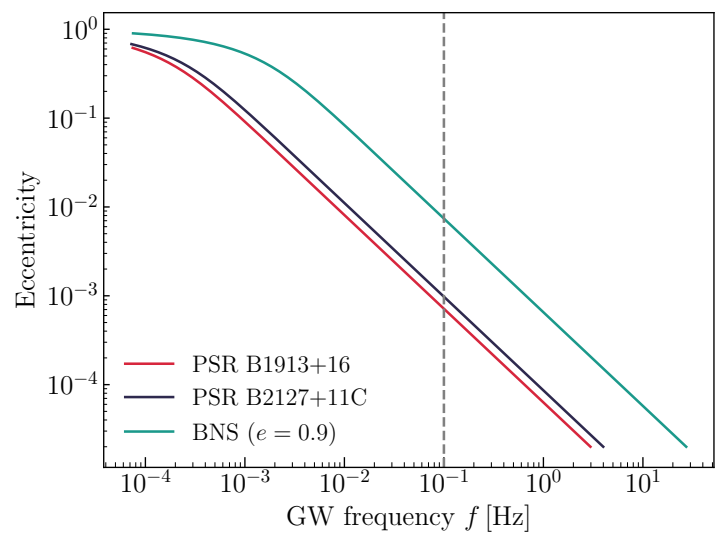

FIG. 1. The evolution of eccentricity with GW frequency for three BNS systems [56].

property of BNSs and simulate the possible BNS systems to be detected by different GW observatories. Then, the distributions of bounds on the dipole radiation parameter are obtained from the ensemble of BNSs. Finally, our main results and discussions are given in Secs. V and VI, respectively. Throughout the paper, we use the geometrized unit where $G=c=1$.

\section{DETECTORS AND SIGNALS}

GW observatories with a lower accessible frequency are more sensitive to the $-1 \mathrm{PN}$ dipole radiation. For the current GW detectors, AdvLIGO, AdV, and KAGRA, their low frequencies are $\gtrsim 10 \mathrm{~Hz}$ [29]. Over the coming decades, they will be upgraded to be more sensitive at low frequency. By 2025, the LIGO detectors will be upgraded to A+LIGO [29]. In the late 2020s, they will be upgraded further to Voyager [54]. In the future, the next-generation ground-based GW detectors, $\mathrm{CE}$ and ET, will further improve their sensitivities. They are roughly an order of magnitude more sensitive than AdvLIGO. CE will be realized in two stages: CE-1 in 2030s and CE-2 in 2040s [32, 33]. ET can extend the accessible frequency down to $\sim 1 \mathrm{~Hz}$. The design sensitivity of ET has been updated a few times, and the latest configuration is known as "ET-D" [34].

For the atom-interferometer detector ZAIGA, its lower accessible frequency is about $0.1 \mathrm{~Hz}$. ZAIGA has two designs, the conservative ZAIGA and the optimal ZAIGA that uses the same Newtonian noise subtraction as ET [46]. In our work, we use the optimal one. In addition, we notice that Chaibi et al. [53] proposed a method of exploiting the Newtonian noises' correlation with an array of long baseline atom-interferometer gradiometers. It is possible to achieve a tenfold or more Newtonian noise rejection. Therefore, we further assume an imaginary detector, that we call $\mathrm{Z}+$, whose strain sensitivity is improved by ten times comparing to ZAIGA. We stress that it is an optimal assumption for now and we use it only for illustrative purposes.

In a summary, we use the following sets of ground-based detectors to constrain dipole radiation: (i) laser-interferometer GW detectors, AdvLIGO at its design sensitivity, A+LIGO, Voyager, CE-1, CE-2, and ET-D, and (ii) ground-based atominterferometer GW detectors, ZAIGA and Z+. These detectors consist of two different types of shapes, the right-angled shape, and the triangle shape. For a triangle detector $\alpha$, there is an additional effective form factor $f_{\alpha}=\sqrt{3} / 2$ compared to an equivalent right-angled detector. Besides, a triangle detector is usually equivalent to two independent detectors. ${ }^{3}$ For a uniform treatment in the calculation, we absorb the form factor, $f_{\alpha}$, as well as its equivalent number of detectors, $n_{\alpha}$, into an effective power sensitivity density, $S_{n, \alpha}^{\text {eff }}{ }^{4}$ Thus, for a detector $\alpha$, we have

$$
S_{n, \alpha}^{\mathrm{eff}}=\frac{1}{f_{\alpha}^{2} n_{\alpha}} S_{n, \alpha}
$$

For different detectors we use, we list their relevant configurations, frequency range $\left[f_{\min }, f_{\max }\right]$, form factor $f_{\alpha}$, equivalent detector number $n_{\alpha}$, and the scheduled epochs when those detectors are to be operational in Table I.

For the BNS waveform, we use the inspiral-mergerringdown phenomenological frequency-domain GW waveform, the so-called IMRPhenomD waveform [59, 60]. A complete GW response for a detector is $\widetilde{h}(f)=F^{+} \widetilde{h}_{+}(f)+$ $F^{\times} \widetilde{h}_{\times}(f)$, where $F^{+, \times}$are the detector's "+/×" component pattern functions related to the sky location $(\theta, \phi)$, and the polarization angle $\psi$ of the GWs. The strain $\widetilde{h}_{+, \times}$can be obtained from the IMRPhenomD model. The difference between BNSs and BBHs in GW waveform mainly comes from the merger and ringdown parts induced by BNSs' matter effects [20]. It is highly related to the BNSs' equation of state. However, for BNSs the frequencies corresponding to the merger and ringdown are too high to contribute to the dipole radiation test in a noticeable way. Therefore, although the IMRPhenomD waveform is built for BBHs, we consider it sufficiently accurate to be used to estimate the dipole radiation parameter for BNSs.

The IMRPhenomD waveform assumes quasi-circular inspirals. According to the formation mechanism of BNSs [61], there could be a significant fraction of eccentric BNS binaries. If the orbital eccentricity of a BNS is non-negligible, the GW amplitudes will split into harmonics with comparable strength, and thus the IMRPhenomD waveform will not be suitable [62]. To explore the effects from eccentricity, we illustrate the evolution of eccentricity with GW frequency for three BNS systems in Fig. 1. Two of them, PSRs B1913+16 [63] and B2127+11C [64], have been long-time monitored by radio telescopes. We can take PSR B1913+16 as an example. Although the eccentricity of PSR B1913+16 is currently $e=0.617$ (the orbital period is 7.75 hours), it will have an eccentricity $e<10^{-3}$ when its GW frequency $f=0.1 \mathrm{~Hz}$. Furthermore, even if we consider a BNS system

\footnotetext{
${ }^{3}$ Note that a triangle ET is equivalent to three independent right-angled detectors because of its specific design $[34,58]$.

${ }^{4}$ For a right-angled detector $\alpha$, we simply have $f_{\alpha}=1$ and $n_{\alpha}$ equals to their actual number of detectors.
} 
TABLE I. Relevant parameters for eight ground-based GW detectors in our study. Note that, the strain sensitivity $\sqrt{S_{n}}$ of the imaginary upgrade of ZAIGA, Z+, is improved by an order of magnitude compared to that of ZAIGA; therefore, we have $S_{n, \mathrm{Z}+}=S_{n, \mathrm{ZAIGA}} / 100$.

\begin{tabular}{|c|c|c|c|c|c|c|c|}
\hline Detector & $f_{\min }(\mathrm{Hz})$ & $f_{\max }(\mathrm{Hz})$ & Shape & $f_{\alpha}$ & $n_{\alpha}$ & $S_{n, \alpha}$ & Schedule \\
\hline AdvLIGO & 5 & 5000 & Right-angled & 1 & 2 & LIGO document ${ }^{\mathrm{a}}$ & O4 [29] \\
\hline $\mathrm{A}+\mathrm{LIGO}$ & 5 & 5000 & Right-angled & 1 & 2 & LIGO document $^{\mathrm{b}}$ & Вy 2025 [29] \\
\hline Voyager & 5 & 10000 & Right-angled & 1 & 2 & LIGO document ${ }^{\mathrm{c}}$ & Late 2020s [54] \\
\hline CE-1 & 3 & 10000 & Right-angled & 1 & 2 & Refs. [32, 33] & 2030s [32] \\
\hline CE-2 & 3 & 10000 & Right-angled & 1 & 2 & Refs. [32, 33] & 2040s [32] \\
\hline ET-D & 1 & 10000 & Triangle & $\sqrt{3} / 2$ & 3 & Ref. [34] & Mid 2030s [55] \\
\hline ZAIGA & 0.1 & 10 & Triangle & $\sqrt{3} / 2$ & 2 & Ref. [46] & - \\
\hline $\mathrm{Z}+$ & 0.1 & 10 & Triangle & $\sqrt{3} / 2$ & 2 & $S_{n, \mathrm{ZAIGA}} / 100$ & - \\
\hline
\end{tabular}

a https://dcc.ligo.org/LIGO-T1800044/public

b https://dcc.ligo.org/LIGO-T1800042/public

${ }^{c}$ https://dcc.ligo.org/LIGO-T1500293/public

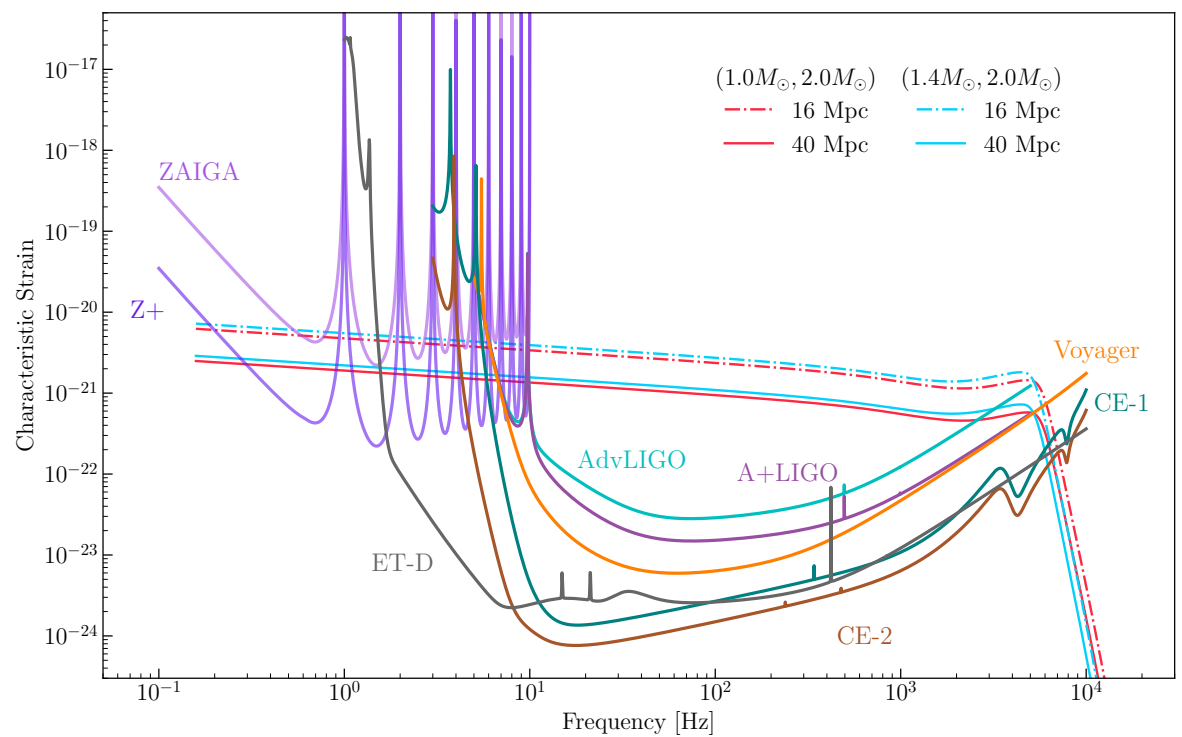

FIG. 2. Noise curves for different detectors and characteristic strains for some example BNS systems. Note that we have taken account of the form factor and the equivalent number of detectors using $S_{n}^{\text {eff }}$ in Eq. (1). Thus the SNR is related to the area between signal and noise curves [57]. The GW waveform used for BNSs is the non-precessing BBH IMRPhenomD model as the high-frequency part has a minor effect in our dipole-radiation study.

"BNS $(e=0.9)$ ", a hypothetical BNS with an orbital period $P_{b}=8.0$ hours and an extreme eccentricity $e=0.9$, the eccentricity at $f \sim 0.1 \mathrm{~Hz}$ is tiny enough to be neglected. In addition, we calculate the number of GW inspiral cycles contributed by eccentricity, $\Delta N_{\mathrm{GW}}^{\mathrm{ecc}}=-7 \times 10^{6} e_{0}^{2}$ where $e_{0}$ is the eccentricity at $f=0.1 \mathrm{~Hz}[65]$. We find $\Delta N_{\mathrm{GW}}^{\mathrm{ecc}}$ is quite small relative to the Newtonian contribution $N_{\mathrm{GW}}^{\mathrm{Newt}} \sim 1 \times 10^{7}$. Therefore, the orbital eccentricity can be assumed to be zero in our consideration.

The effective detector noise amplitude is $h_{n}=\sqrt{f S_{n}^{\text {eff }}}$ and the characteristic strain amplitudes of GWs are $h_{c}=$ $2 f|\tilde{h}|$ [57]. In Fig. 2, we illustrate the $h_{n}$ for different groundbased detectors. In addition, we plot the $(\theta, \phi, \psi)$-averaged and face-on $(\iota=0)$ characteristic strain $h_{c}$ of GWs from typical
BNS systems.

The signal-to-noise ratio (SNR) $\rho$ for a detector can be expressed as $\rho=(\widetilde{h}(f) \mid \widetilde{h}(f))^{1 / 2}$, where the noise-weighted inner product is defined via,

$$
(\widetilde{A}(f) \mid \widetilde{B}(f)) \equiv 2 \int_{f_{\min }}^{f_{\max }} \frac{\widetilde{A^{\star}}(f) \widetilde{B}(f)+\widetilde{A}(f) \widetilde{B}^{\star}(f)}{S_{n}^{\text {eff }}} \mathrm{d} f .
$$

In Fig. 2, the area between $h_{n}$ and $h_{c}$ curves is proportional to the SNR [57]. As we can see, a $\left(1.4 M_{\odot}, 2.0 M_{\odot}\right)$ BNS at a luminosity distance $D_{\mathrm{L}}=40 \mathrm{Mpc}$ (at the same distance as the first detected BNS inspiral GW170817) can hardly be detected by ZAIGA alone as it has a too small SNR. Only very close BNSs are detectable by ZAIGA-like detectors.

We illustrate the range of luminosity distances for equal- 


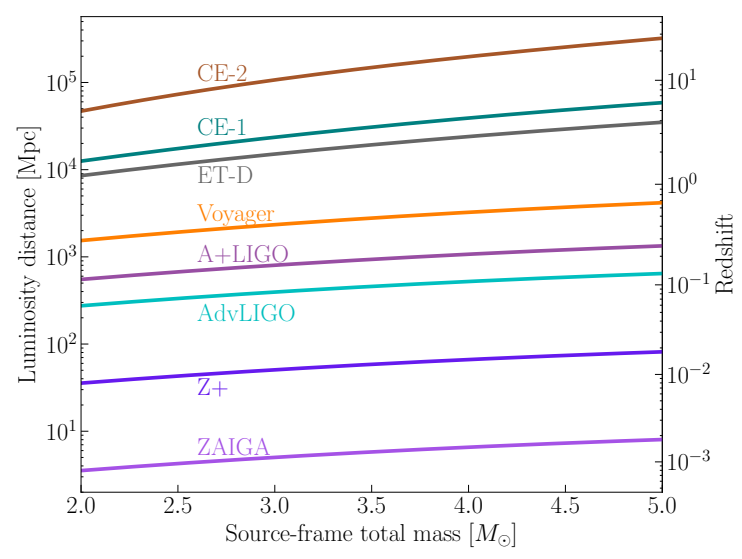

FIG. 3. The luminosity distance (left) of equal-mass face-on BNSs and the corresponding redshift (right) with a $\operatorname{SNR} \rho=8$ for groundbased GW detectors. We have averaged the sky location and GW polarization angles.

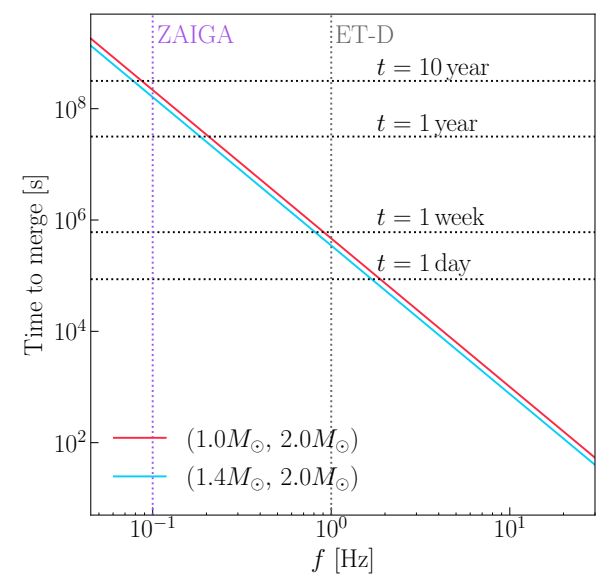

FIG. 4. Time to coalescence for two example BNS sources. The vertical dotted lines correspond to the lower frequency of ZAIGA/Z+ and ET-D.

mass face-on BNSs with different detectors assuming a threshold SNR $\rho=8$ in Fig. 3. In the figure, the sky location of binaries and $\mathrm{GW}$ polarization angle have been averaged. In addition, we adopt a cosmology with the matter density $\Omega_{\mathrm{M}} \simeq$ 0.315 , the dark energy density $\Omega_{\Lambda}=1-\Omega_{\mathrm{M}} \simeq 0.685$, and the Hubble constant at today $H_{0} \simeq 67.4 \mathrm{~km} \mathrm{~s}^{-1} \mathrm{Mpc}^{-1}$ [66] to convert between the source redshift $z$ and its luminosity distance $D_{\mathrm{L}}$, where the luminosity distance as a function of redshift $z$ is given by $D_{\mathrm{L}}(z)=(1+z) \int_{0}^{z} \mathrm{~d} z^{\prime} / H\left(z^{\prime}\right)$, with $H(z) \equiv H_{0} \sqrt{\Omega_{\mathrm{M}}(1+z)^{3}+\Omega_{\Lambda}}$. As we can see in Fig. 3, ZAIGA can hardly detect GWs alone from BNSs whose luminosity distances are larger than $10 \mathrm{Mpc}$. For $\mathrm{Z}+$, its reach to BNSs is less than $100 \mathrm{Mpc}$, but it is already within the astrophysically interesting range. ${ }^{5}$ Among the detectors we

\footnotetext{
${ }^{5}$ Note that the first detected BNS inspiral, GW170817, is certainly within Z+'s reach.
}

use, CE-2 can detect the furthest BNSs, to a distance as far as $\sim 100 \mathrm{Gpc}$.

The frequency of GW, $f$, evolves at the Newtonian order via $\dot{f} / f=96(\pi \mathcal{M} f)^{8 / 3} / 5 \mathcal{M}$, for a binary system, where $\mathcal{M}=\left(m_{1} m_{2}\right)^{3 / 5} /\left(m_{1}+m_{2}\right)^{1 / 5}$ is the redshifted chirp mass with redshifted component masses $m_{1}$ and $m_{2}$. Integrating it from an initial frequency $f_{\text {in }}$, one obtains the time to coalescence, $\Delta \tau\left(f_{\text {in }}\right) \approx 5 \mathcal{M}\left(\pi \mathcal{M} f_{\text {in }}\right)^{-8 / 3} / 256$. We show the time to coalescence at $z=0$ for typical BNSs in Fig. 4. As we can see, for a $\left(1.0 M_{\odot}, 2.0 M_{\odot}\right)$ binary system, the GW signals stay for a very long time. For ET, it can last a week before its coalescence. For ZAIGA/Z+, we can record the GW signal for more than 1 year. This shows exactly the point that a lower accessible frequency can be advantageous to test the negative $\mathrm{PN}$ effects in BNS systems, such as the dipole radiation.

\section{DIPOLE RADIATION IN BNS SYSTEMS}

One of the earliest motivations to test dipole radiation comes from the Jordan-Fierz-Brans-Dicke (JFBD) theory [9]. This theory adds a scalar degree of freedom in addition to the metric field in GR. Famous extensions include the DamourEsposito-Farèse theory [52, 67], which predicts strong-field scalarization for NSs and has been tested by a couple of binary pulsars $[13,14,16-18]$. The constraints on dipole radiation from binary pulsars are rather tight now in the quasi-stationary strong-field regime [13]. For the highly-dynamical strongfield regime, compact binary coalescences become one of the most powerful testbeds. For example, certain kind of theories (e.g. with a scalar Gauss-Bonnet coupling term [51]) predict scalar hairs for BHs, and the dipole radiation in these theories can be constrained with BBH systems [12, 26, 43]. The specifics in the underlying gravity theory determine whether NSs or BHs get scalarized. In this paper, with the DamourEsposito-Farèse gravity $[52,67]$ kept in mind as a prototype theory, we use GWs from BNS systems to test the dipole radiation. But the analysis can be extended to other scenarios as well when needed.

The extra scalar field not only causes an additional gravitational binding energy shift for the orbit but also enhances the energy flux via extra dipole radiations. Generically, the modified GW flux can be parameterized as [26]

$$
\dot{E}^{\mathrm{GW}}=\dot{E}^{\mathrm{GR}}\left[1+B\left(\frac{m}{r}\right)^{-1}\right],
$$

where $\dot{E}^{\mathrm{GR}}$ is the GW flux derived from GR's quadrupolar radiation, $m$ and $r$ are respectively the total mass and the orbital separation of the binary, and the theory-dependent parameter $B$ describes the strength of the additional dipole radiation. Compared to the quadrupolar radiation in GR, dipole radiation is enhanced by a factor of $\sim(\mathrm{m} / r)^{-1} \sim(v / c)^{-2}$, where $v$ is the characteristic relative speed in the binary. As said before, the dipole emission is a $-1 \mathrm{PN}$ effect, and dominates relatively at a larger separation $r$, or at a lower GW frequency. For JFBDlike scalar-tensor theories, $B$ equals to $(5 / 96)|\Delta \alpha|^{2}$, where $\Delta \alpha$ describes the difference between the effective scalar couplings of two objects in the binary $[52,68]$. 
To incorporate the effects from dipole radiation into GW waveform, we use the ppE framework [68, 69]. This framework generically parameterizes GW waveform's deviations from the GR prediction. The GW waveform for a compact binary inspiral in the ppE framework is given by,

$$
\widetilde{h}(f)=\widetilde{h}^{\mathrm{GR}}(f) \cdot\left(1+\alpha u^{a}\right) e^{\mathrm{i} \beta u^{b}},
$$

where $u \equiv \pi \mathcal{M} f$, and $\widetilde{h}^{\mathrm{GR}}(f)$ is the waveform in GR. The amplitude and phase of GW waveform are corrected by the ppE parameters $(a, \alpha)$ and $(b, \beta)$ respectively $[68,69]$. In particular, Eq. (4) reduces to the waveform in GR with $\alpha=\beta=0$. For theories with dipole radiation, the general modification to the inspiral phase is given by $b=-7 / 3$, and $\beta$ is connected to the dipole radiation parameter $B$ in Eq. (3) via $-(3 / 224) \eta^{2 / 5} B$. Note that, the non-GR corrections for the GW amplitude are less important in general, as the matched filtering is more sensitive to the phase evolution. Having neglected the matter effects at high frequency in the merger-ringdown stages, we use the IMRPhenomD BBH waveform to represent $\widetilde{h}^{\mathrm{GR}}(f)$ in Eq. (4). Also, when we consider the ppE modified waveform, it is sufficient to only include the inspiral phase corrections in our current problem.

\section{TESTING DIPOLE RADIATION WITH BNS POPULATIONS}

In our test, the dipole radiation is parameterized by the parameter $B$ in Eq. (3). Before investigating the bounds on the parameter $B$ with the Fisher information matrix (FIM) method, we can use the number of GW inspiral cycles, $N_{\mathrm{GW}}$, for a rough estimate of bounds on $B$ [70]. Here, we take a typical BNS system as an example. For a $\left(1.4 M_{\odot}, 1.4 M_{\odot}\right)$ BNS system detected by ZAIGA/Z+, the number of GW inspiral cycles contributed by dipole radiation is $\Delta N_{\mathrm{GW}}^{\text {dipole }} \sim$ $-2 \times 10^{10} B$. When we set $\left|\Delta N_{\mathrm{GW}}^{\mathrm{dipole}}\right| \lesssim 1$, we can obtain a rough estimation, $B \lesssim 5 \times 10^{-11}$. In addition, we use another method from Ref. [26], using $\left|(\Delta t)_{\mathrm{GR}}-(\Delta t)_{\text {non-GR }}\right|$ to roughly bound $B$. The result is consistent with the constraint from $\left|\Delta N_{\mathrm{GW}}^{\text {dipole }}\right|<1$. Note that, these simple methods above only involve parameter $B$, ignoring correlations between $B$ and other parameters. For more complete analyses, we will use the FIM for a more reasonable estimation. ${ }^{6}$

The FIM is constructed as usual from the frequency-domain waveform $\widetilde{h}(f)$,

$$
\Gamma_{i j} \equiv\left(\frac{\partial \widetilde{h}(f)}{\partial \xi_{i}} \mid \frac{\partial \widetilde{h}(f)}{\partial \xi_{j}}\right) .
$$

Here, the parameter $\xi_{i} \in\left\{\ln \mathcal{A}, \ln \mathcal{M}, \ln \eta, \chi_{1}, \chi_{2}, \phi_{c}, t_{c}, B\right\}$ where $\mathcal{A}$ is the GW amplitude, $\eta=m_{1} m_{2} /\left(m_{1}+m_{2}\right)^{2}$ is the

\footnotetext{
${ }^{6}$ Comparing $\Delta N_{\mathrm{GW}}^{\mathrm{dipole}}$ and $\Delta N_{\mathrm{GW}}^{\mathrm{ecc}}$, it might be possible to discuss whether one can neglect the eccentricity in ppE waveforms though this is a simple analysis. For a better analysis, one might apply the same analysis in Ref. [71] to eccentric ppE waveforms based on Ref. [72].
}

symmetric mass ratio of the binary, $\phi_{c}$ and $t_{c}$ are the phase and time at coalescence respectively, and $\chi_{1,2}$ is the dimensionless aligned spin components of two NSs. For a parameter $\xi_{i}$, as per the Cramér-Rao inequality, a lower bound on its standard deviation expected from an experiment can be placed with $\sigma\left(\xi_{i}\right) \geq \sqrt{\left(\Gamma^{-1}\right)_{i i}}[73-75]$. We refer the readers to Appendix A for a brief explanation of the FIM method.

To have a statistically sound estimate, we consider the population property of BNSs and simulate the possible BNS systems across the cosmos. First, we denote the BNS merger rate per unit proper time per unit co-moving volume at $z=0$ by $\mathcal{R}_{0}$. It is convenient to have BNS merger rate at redshift $z$ using,

$$
\mathcal{R}(z)=\mathcal{R}_{0} r(z) .
$$

Here, the merger rate's time evolution is encapsulated in $r(z)$. We use the same piecewise linear fitting to $r(z)$ as that in Refs. [76, 77]. In that way, $r(z)$ can be expressed using the following piecewise function,

$$
r(z)= \begin{cases}1+2 z, & z<1, \\ \frac{3}{4}(5-z), & 1 \leq z \leq 5, \\ 0, & z>5\end{cases}
$$

This is a rough approximation to the realistic BNS population, but suffices for our study here.

Over a fixed observing duration $\Delta T$, the total number of BNS merger events, $N_{\text {event }}$, detected by a single detector is,

$$
N_{\text {event }}=\Delta T \times \int_{0}^{z_{\text {th }}} \frac{4 \pi D_{\mathrm{COM}}^{2}(z) \mathcal{R}(z)}{(1+z) H(z)} \mathrm{d} z
$$

where $z_{\text {th }}$ is the redshift corresponding to response distance for a detector [78] and $D_{\mathrm{COM}}(z)=D_{\mathrm{L}}(z) /(1+z)$ is the co-moving distance to the BNS system. Here, for each detector we set $\Delta T=1$ year, as a conservative operational duration, to derive the total numbers of BNS merger events. ${ }^{7}$

In reality, many BNSs will be detected by the aforementioned GW detectors except ZAIGA. Some of these BNSs will be of low SNRs, while the others are rather loud. Depending on the specific systems' physical parameters, these BNSs will provide different limits on the dipole radiation parameter $B$. The ultimate constraint is to be dominated by the best limit, or a combination of a few best ones. Therefore, in this paper, we focus on one or a few best bounds from the BNS population. We randomly generate BNS populations from an underlying population model and calculate the optimal uncertainty of $B$ in each population. Such a treatment differs from some earlier studies but is close to reality when a test of dipole radiation is to be carried out.

For some detectors (e.g., Voyager, CE, and ET), their detection ranges, denoted by the redshift $z_{\text {th }}$, are very large. The

\footnotetext{
${ }^{7}$ Correspondingly, for ZAIGA/Z+, considering the operational duration, we actually use the frequency one year before coalescence, $f_{\min }=f_{1 \mathrm{yr}}$, in Eq. (2). For a $\left(1.4 M_{\odot}, 1.4 M_{\odot}\right)$ BNS system at $z=0, f_{1 \mathrm{yr}} \sim 0.2 \mathrm{~Hz}$.
} 
computational cost for the whole population is expensive. As we have explained, the optimal constraints are to be obtained from the closer binary systems to us. Therefore, instead of using the threshold redshift $z_{\text {th }}$ in Eq. (8), we use $z=0.5$ for Voyager, CE, and ET detectors. It is enough for calculating the optimal bounds on $B$ in our study. For ZAIGA, its $z_{\text {th }}$ is too small to detect a realistic BNS system unless we are very lucky. Therefore, we set an over-optimal $z_{\text {th }}=0.02$ for ZAIGA, which is the same value as that from $Z+$. Note that, the sensitivity of ZAIGA is still worse than that of $\mathrm{Z}+$. This approach only ensures that ZAIGA will have some (lowSNR) events to be analyzed; see more details in the following text about the usage of ZAIGA with other ground-based laser interferometers. The threshold redshift $z_{\text {th }}$ that we use in the simulation and the corresponding total numbers of BNS merger events per year, $N_{\text {event }}$, are given in Table II.

In the simulation, we generate $N_{\text {event }}$ possible BNS systems within one-year time according to Eq. (8). Meanwhile, the angle parameters for the sky location, $\cos \theta$ and $\phi$, are generated randomly from $[-1,1]$ and $[0,2 \pi)$, respectively. The GW polarization angle $\psi$ is uniform in $[0,2 \pi)$ and the inclination angle $\cos \iota$ is generated uniformly in $[-1,1]$. The sourceframe masses of binaries are obtained in Gaussian distribution $\mathcal{N}\left(1.35 M_{\odot},\left(0.09 M_{\odot}\right)^{2}\right)[7,79]$. A "low spin" BNS population, where the aligned spin components $\chi_{1,2}$ are uniform in $[-0.05,0.05]$, is assumed in our computation. A recent merger rate of BNS, $\mathcal{R}_{0}=1210 \mathrm{Gpc}^{-3}$ year ${ }^{-1}$, is used in the simulation [7].

According to Table II, we can see that at least thousands of BNS systems could be detected in AdvLIGO, A+LIGO, Voyage, CE and ET. However, only the few ones that give the best constraints on dipole radiation contribute to the final bounds on $B$ in our simulation, while the majority of the remaining BNS samples hardly contribute. As we know, for a given detector, the FIM is roughly proportional to the square of SNR $\rho^{2} \propto \mathcal{A}^{2}$, according to Eq. (5). In this way, we expect the bounds on the dipole radiation parameter $\sigma(B) \propto \rho^{-1} \propto \mathcal{A}^{-1}$ in a rough manner. Therefore, to simplify the computation further, we construct an effective amplitude in proportional to the original $\mathcal{A}$,

$$
\mathrm{A}=\left[\left(1+\cos ^{2} \iota\right)^{2} F_{+}^{2}+4 \cos ^{2} \iota F_{\times}^{2}\right]^{1 / 2} \frac{\mathcal{M}^{5 / 6}}{D_{\mathrm{L}}},
$$

to reduce the total number of BNS mergers in the FIM calculation. We use A to get the loudest BNS systems in advance. Then, instead of using all possible BNS mergers, only the loudest BNS systems associated with relatively larger A's are considered in our FIM calculation. For a detector, we choose the total number of the loudest BNS systems to be $N=\min \left(N_{\text {event }}, N_{\text {cut }}\right)$. Here, we set $N_{\text {cut }}=30 .{ }^{8}$ In other words, if $N_{\text {event }}>N_{\text {cut }}$, we will only use the top $N_{\text {cut }}$ BNS systems corresponding to large A's. According to the formula for

\footnotetext{
${ }^{8}$ For a negative PN effect (here, dipole radiation effect), the combined bounds are usually from a few tightest bounds. Our setting $N_{\text {cut }}=30$ is consistent with the results of Ref. [80].
}

combination (see below), it is enough to obtain the constraints from those BNS systems. We record the tightest constraint, $\sigma_{\text {opt }}(B)$. In addition, the combined constraint from $N$ events, denoted as $\sigma_{\text {com }}(B)$, is obtained via,

$$
\frac{1}{\sigma_{\mathrm{com}}^{2}(B)}=\sum_{i=1}^{N} \frac{1}{\sigma_{i}^{2}(B)} .
$$

We repeat simulations with random samples 1000 times for each $\mathrm{GW}$ detector. At the end, we collect results of $\sigma(B)$. The distributions of 1000 "optimal" bounds, $\sigma_{\text {opt }}(B)$, and 1000 "combined" bounds, $\sigma_{\text {com }}(B)$, can be obtained from the above simulations. Moreover, to investigate how laser interferometers and atom interferometers will complement each other in testing the dipole radiation in BNS systems, we take two scenarios as follows,

- Scenario (I): investigating the bounds on the dipole radiation parameter from individual ground-based detectors;

- Scenario (II): investigating the joint bounds on $B$ by strategically combining atom interferometers, ZAIGA/Z+, with laser interferometers.

In different scenarios, we perform the parameter estimation with FIM and obtain the desired bounds, which are presented in the next section.

\section{PROJECTED CONSTRAINTS ON DIPOLE RADIATION}

From the setup of simulations in the last section, we obtain the distributions of the parameter $\sigma(B)$, including $\sigma_{\text {opt }}(B)$ and $\sigma_{\text {com }}(B)$. They are illustrated in Figs. 5 and 6, and will be explained further in the following. For each detector, the median values of $\sigma(B)$ from the distributions are collected in Table II for references.

In Fig. 5, we illustrate the constraints on $B$, namely $\sigma(B)$, from individual ground-based GW detectors. It is evident that, for ground-based laser-interferometer detectors, ET-D can provide the tightest constraint on the parameter $B$, where $\sigma_{\text {com }}(B)$ is about $9 \times 10^{-10}$. It is due to its good sensitivity and its low accessible frequency, starting from $1 \mathrm{~Hz}$ (see Table I). As for atom-interferometer detectors, benefiting from their lower accessible frequency than that of laser-interferometer detectors, the bounds on $B$ can reach $\sigma(B) \lesssim 10^{-8}$ in ZAIGA and $\lesssim 10^{-9}$ in $\mathrm{Z}+$. However, the SNRs in these two detectors are rather low, namely $\rho_{\text {mean }} \sim 0.3$ for ZAIGA and $\rho_{\text {mean }} \sim 3$ for $\mathrm{Z}+$. These low SNRs in ZAIGA and Z+ will prevent us from positively detecting the BNS signals, and thus the bounds on $B$ from FIM are meaningless when only the atom interferometers are considered [75].

We expect that technically speaking, although with very low SNRs for signals, ZAIGA/Z+ can record all the strain data for BNS mergers whatsoever. These "GW signals" will be buried deep in noises. Late in the frequency evolution of these signals, these BNSs will positively trigger laserinterferometer detectors with enough SNRs. Then, we can 
TABLE II. Relevant parameters for the simulation of BNS populations and the median bounds on the relevant parameters. For $N_{\text {event }}$ we list the number of BNSs that have $\rho \gtrsim 8$. Note that, “*” stands for the bounds on parameters using an 'improper' FIM, and the results are roughly suitable only for the order-of-magnitude estimates.

\begin{tabular}{llllllll}
\hline \hline Detector & $z_{\text {th }}$ & $N_{\text {event }}$ & $\begin{array}{l}\sigma_{\text {opt }}(B) \\
(\text { median })\end{array}$ & $\begin{array}{l}\sigma_{\text {com }}(B) \\
(\text { median })\end{array}$ & $\begin{array}{l}\sigma_{\text {opt }}(\mathcal{M}) / \mathcal{M} \\
(\text { median })\end{array}$ & $\begin{array}{l}\sigma_{\text {opt }}(\eta) / \eta \\
(\text { median })\end{array}$ & $\begin{array}{l}\sigma_{\text {opt }}\left(t_{c}\right)[\mathrm{s}] \\
(\text { median })\end{array}$ \\
\hline ZAIGA & $<0.02$ & $<4$ & $1.3 \times 10^{-8 *}$ & $9.8 \times 10^{-9} *$ & $1.2 \times 10^{-5 *}$ & $4.0 \times 10^{-3 *}$ & $6.4 \times 10^{-1 *}$ \\
Z+ & $\lesssim 0.02$ & $\lesssim 4$ & $1.2 \times 10^{-9 *}$ & $9.4 \times 10^{-10 *}$ & $1.1 \times 10^{-6 *}$ & $3.9 \times 10^{-4 *}$ & $6.0 \times 10^{-2 *}$ \\
AdvLIGO & $\lesssim 0.2$ & $\sim 35$ & $7.2 \times 10^{-7}$ & $3.0 \times 10^{-7}$ & $5.8 \times 10^{-5}$ & $8.9 \times 10^{-3}$ & $7.1 \times 10^{-5}$ \\
A+LIGO & $\lesssim 0.3$ & $\sim 280$ & $4.8 \times 10^{-7}$ & $2.0 \times 10^{-7}$ & $3.5 \times 10^{-5}$ & $4.6 \times 10^{-3}$ & $3.4 \times 10^{-5}$ \\
Voyager & $\sim 0.5$ & $\sim 5600$ & $2.1 \times 10^{-7}$ & $8.9 \times 10^{-8}$ & $1.6 \times 10^{-5}$ & $2.0 \times 10^{-3}$ & $2.3 \times 10^{-5}$ \\
CE-1 & $>0.5$ & $>47551$ & $2.8 \times 10^{-8}$ & $1.2 \times 10^{-8}$ & $2.2 \times 10^{-6}$ & $1.9 \times 10^{-4}$ & $5.1 \times 10^{-6}$ \\
CE-2 & $>0.5$ & $>47551$ & $8.4 \times 10^{-9}$ & $3.5 \times 10^{-9}$ & $7.8 \times 10^{-7}$ & $1.0 \times 10^{-4}$ & $2.7 \times 10^{-6}$ \\
ET-D & $>0.5$ & $>47551$ & $2.1 \times 10^{-9}$ & $8.7 \times 10^{-10}$ & $3.3 \times 10^{-7}$ & $5.6 \times 10^{-5}$ & $4.2 \times 10^{-6}$ \\
\hline
\end{tabular}

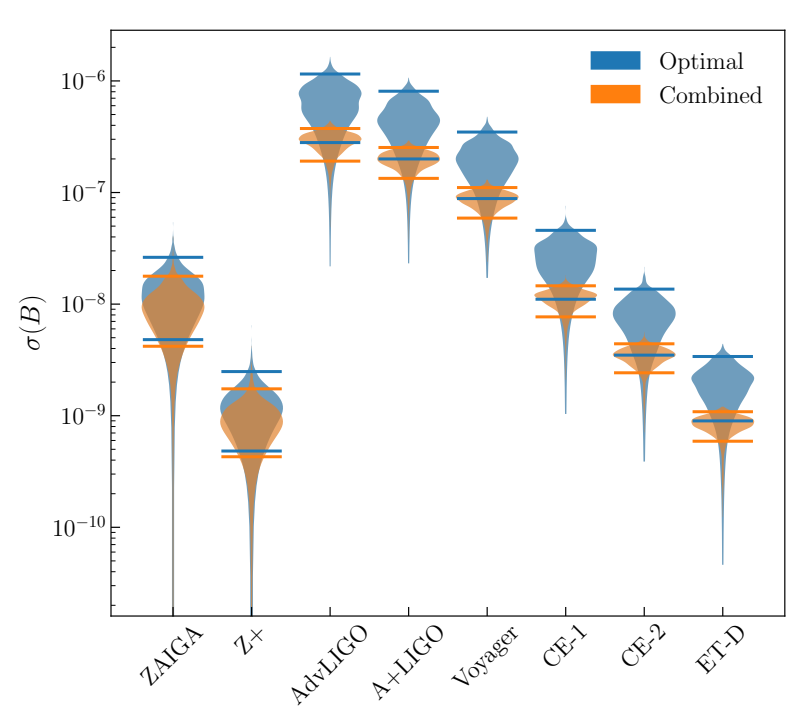

FIG. 5. The distribution of $\sigma_{\text {opt }}(B)$ (blue) and $\sigma_{\text {com }}(B)$ (orange) from eight ground-based detectors in Scenario (I). The upper and lower bars show the values at $5 \%$ and $95 \%$ confidence levels.

confidently assume that these GW signals are also recorded in atom-interferometer detectors, though not being loud enough to trigger a detection. With this assumption, we can combine data from atom interferometers and laser interferometers. Such a combination is not useful to obtain a larger SNR but will put tight constraints on negative PN terms, as they are so sensitively dependent on the low-frequency data. Thus, a tighter constraint on $B$ can be derived from the recorded ZAIGA/Z+ data with the help of other detectors. To our knowledge such kind of combination is firstly proposed in literature. The details certainly deserve further investigation.

With the above guidelines in mind, in Fig. 6, we focus on the joint constraints from ZAIGA/Z+ together with the other six laser-interferometer detectors. As we can see, with the contributions from $\mathrm{ZAIGA} / \mathrm{Z}+$, the joint constraints on $B$ are improved significantly for laser interferometers. Especially, for AdvLIGO, A+LIGO, and Voyager, the joint constraints $\sigma(B)$ can be tightened to $\lesssim 1 \times 10^{-9}$ with ZAIGA. It is im- proved by more than an order of magnitude. It means that, in the future joint observations, AdvLIGO, A+LIGO, Voyager, and CE can help ZAIGA/Z+ to extract BNS parameters, and then ZAIGA/Z+ can help them to constrain the dipole radiation in return. In addition, we also notice that the bounds on dipole radiation from $\mathrm{ZAIGA} / \mathrm{Z}+$ alone are similar to the results from the joint constraints when $\mathrm{ZAIGA} / \mathrm{Z}+$ is combined with other laser-interferometer detectors. Although the constraints from ZAIGA/Z+ alone in Fig. 5 are derived from inappropriate FIM calculation, they still show ZAIGA/Z+'s potential in testing dipole radiation. In this sense, it is easy to understand that in the joint analysis, ZAIGA/Z+ plays the key role in constraining the dipole radiation, albeit with very low SNRs for BNS events. Of course, ZAIGA and Z+ are very hard to properly constrain the dipole radiation as BNSs are hardly detected in these detectors without the help of laserinterferometer detectors. Therefore, it will be of great help to estimate the possible bounds on the dipole radiation by considering the joint observation in the future. As we see from the figure, $\mathrm{Z}+$ can help ET improve the constraint on $B$ from $\sigma(B) \sim 2 \times 10^{-9}$ to $\sigma(B) \sim 1 \times 10^{-10}$. Furthermore, in Fig. 6, it is shown that, compared to ground-based laser interferometers alone, the tighter bounds on $B$ can ultimately reach the levels of $|B| \lesssim 10^{-9}$ with ZAIGA and $|B| \lesssim 10^{-10}$ with Z+ in the joint observations.

Now, we turn to the bounds on the other parameters, such as $\mathcal{M}, \eta$, and $t_{c}$. The constraints from individual $\mathrm{GW}$ detectors and joint observations including ZAIGA/Z+ are shown in Table II and Fig. 7, respectively. In addition to $B$, the bounds on $\mathcal{M}$ and $\eta$ are improved with the help of ZAIGA/Z+, relative to the bounds from laser-interferometers only. The joint constraints on $t_{c}$ are similar to these from laser-interferometers alone. It indicates that ZAIGA/Z+ can hardly improve the sky location in the joint observations.

\section{DISCUSSIONS}

In this paper, we studied the projected bounds from nearfuture ground-based $\mathrm{GW}$ detectors on dipole radiation using a popular parametrization (3) by Barausse et al. [26]. Grav- 


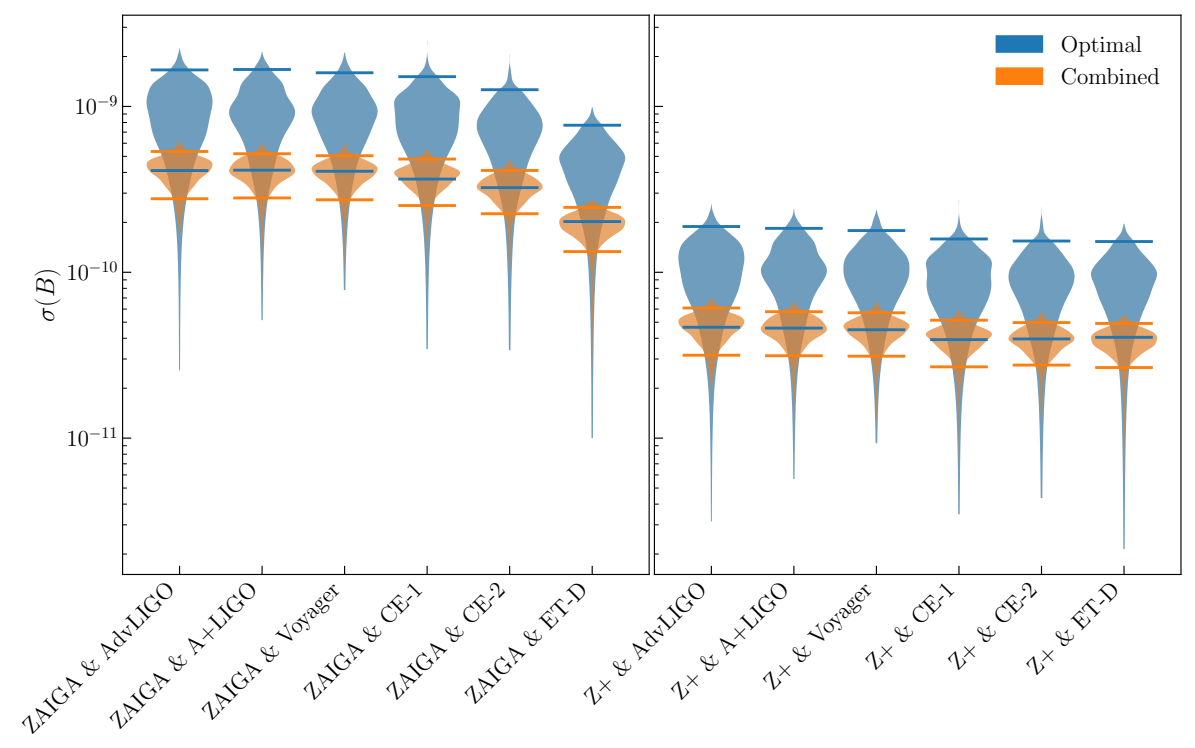

FIG. 6. Same as Fig. 5, but for Scenario (II), where the FIMs of laser interferometers are combined with that of atom interferometers ZAIGA (left) and $\mathrm{Z}+$ (right).

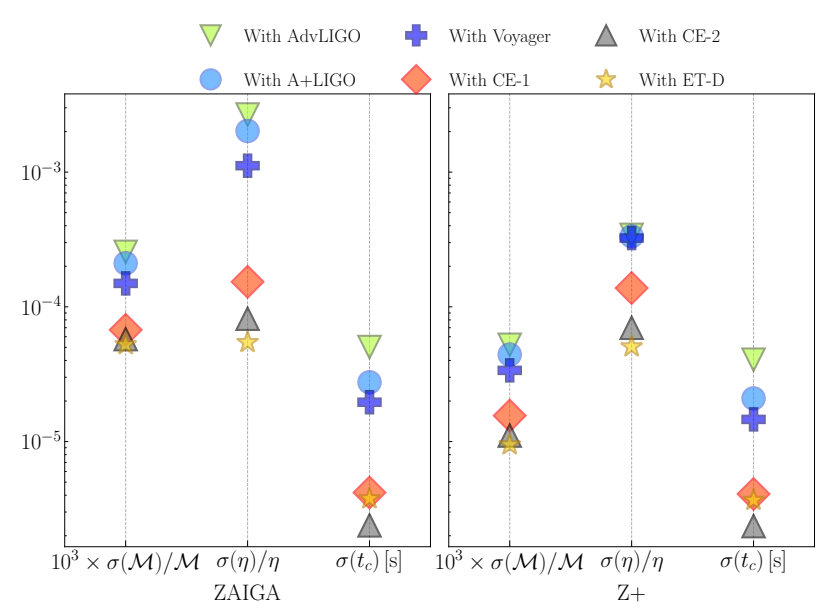

FIG. 7. The median of the joint bounds on other parameters, $\mathcal{M}, \eta$, and $t_{c}$.

itational dipole radiation could come from some alternative theories with additional gravitational degrees of freedom in addition to $g_{\mu v}$ in GR. Our work put the focus on BNS systems. It is relevant to some class of alternative gravity theory, e.g. the Damour-Esposito-Farése theory. We used the modified GW waveforms augmented by dipole radiation in the $\mathrm{ppE}$ framework [68, 69]. Meanwhile, we considered the population property of BNS systems and simulated possible BNS systems to be detected by ground-based laser-interferometer and atom-interferometer detectors. After performing parameter estimation with the FIM technology, we obtained the distribution of bounds on the dipole radiation. To investigate the effects of atom-interferometer detectors (ZAIGA/Z+ in our paper), we compared different scenarios in detail. The main conclusions are summarized in the following. (i) For the atom-interferometer detectors ZAIGA and Z+, due to their limited sensitivity, it is hard to detect a distant BNS system with a high SNR. However, they are still useful in constraining the dipole radiation when combined with the confident detections from other ground-based laser-interferometer detectors. In that way, the constraints on dipole radiation can be tightened significantly in the future.

(ii) In spite of low SNRs, the constraints on $B$ from ZAIGA/Z+ are tighter than most ground-based laser interferometer detectors. The constraints derived from the inappropriate FIM method for ZAIGA/Z+ can be used as an order-of-magnitude estimate. According to the results of Scenario (II) in Sec. IV, where atom interferometers are combined with laser interferometers, ZAIGA/Z+ can help other ground-based detectors get tighter bounds. ${ }^{9}$

(iii) Due to its low accessible frequency and high sensitivity, ET can provide a tight bound on $B$ alone, $\sigma(B) \sim 2 \times$ $10^{-9}$. Even so, the constraint can still be improved to the level of $O\left(10^{-10}\right)$ from its joint observation with $\mathrm{Z}+$.

As summarized above, the atom-interferometer detectors ZAIGA/Z+ may have limited potential to detect a GW from realistic BNSs alone, but they can complement other groundbased laser-interferometer detectors in testing the gravitational dipole radiation from BNS mergers. This is largely due

\footnotetext{
${ }^{9}$ It might be better to use other methods like the Markov-chain Monte Carlo (MCMC) method, see e.g., Ref. [81], to confirm the constraints on $B$ derived from FIM by combining the low SNR waveforms in the atominterferometer band with the waveforms in the laser-interferometer band. This is left for future work because the MCMC method is time-consuming.
} 
to the low accessible frequency for atom interferometers. In the future, we expect the constraints on dipole radiation can be tightened with different types of ground-based GW detectors' complementary roles. The elements crucial to the SEP, which are closely associated with the dipole radiation in strong-field gravity, can be tested further. Meanwhile, as we have shown in this work, probing dipole radiation with BNS systems can be an additional science case for building atom-interferometer detectors like ZAIGA/Z+.

\section{ACKNOWLEDGMENTS}

We thank the anonymous referee for constructive comments that improved the work. We are grateful to Dong-Feng Gao, Huimei Wang, and Ming-Sheng Zhan for helpful discussions. This work was supported by the National Natural Science Foundation of China (11975027, 11991053, 11721303), the National SKA Program of China (2020SKA0120300), the Young Elite Scientists Sponsorship Program by the China Association for Science and Technology (2018QNRC001), and the Max Planck Partner Group Program funded by the Max Planck Society. It was partially supported by the Strategic Priority Research Program of the Chinese Academy of Sciences through the Grant No. XDB23010200, and the Highperformance Computing Platform of Peking University.

\section{Appendix A: Fisher Information Matrix}

In estimation of the parameter set $\boldsymbol{\xi}$, when the SNR is large, the probability $p(\Delta \boldsymbol{\xi})$ is characterized by $[82,83]$,

$$
\ln p(\Delta \xi) \propto-\frac{1}{2} \Gamma_{i j} \Delta \xi_{i} \Delta \xi_{j}
$$

where $\Delta \xi_{i} \equiv \xi_{i}-\hat{\xi}_{i}$, with the maximum-likelihood estimate $\hat{\xi}_{i}$ determined in the matched-filtering method. The variancecovariance matrix for estimating the parameters $\boldsymbol{\xi}$ with a network of detectors is derived by inversing the network FIM, which is obtained by summing the individual Fisher matrices [70, 82],

$$
\Gamma_{i j}=\sum_{\alpha} \Gamma_{i j}^{\alpha}
$$

To calculate the FIM, we need to compute partial derivatives of $\widetilde{h}(f)$ with respect to different parameters. For GW amplitude $\mathcal{A}$, the time at coalescence $t_{c}$, the phase at coalescence $\phi_{c}$, and the dipole parameter $B$, we use the analytical results,

$$
\begin{aligned}
\frac{\partial \widetilde{h}(f)}{\partial \ln \mathcal{A}} & =\widetilde{h}(f) \\
\frac{\partial \widetilde{h}(f)}{\partial t_{c}} & =\mathrm{i} 2 \pi f \widetilde{h}(f), \\
\frac{\partial \widetilde{h}(f)}{\partial \phi_{c}} & =-\mathrm{i} \widetilde{h}(f), \\
\frac{\partial \widetilde{h}(f)}{\partial B} & =-\mathrm{i} \frac{3}{224 \eta}(\pi M f)^{-7 / 3} \widetilde{h}(f) .
\end{aligned}
$$

For the other parameters, including the aligned dimensionless spins, the chirp mass, and the symmetric mass ratio, the partial derivatives are obtained numerically by

$$
\frac{\tilde{\partial h}(f)}{\partial \xi_{i}} \approx \frac{\Delta \widetilde{h}(f)}{\Delta \xi_{i}} \equiv \frac{\widetilde{h}\left(\xi_{i}+\Delta \xi_{i} ; f\right)-\widetilde{h}\left(\xi_{i} ; f\right)}{\Delta \xi_{i}}
$$

[1] C. M. Will, Theory and Experiment in Gravitational Physics (Cambridge University Press, Cambridge, England, 2018).

[2] A. Einstein, Sitzungsber. Preuss. Akad. Wiss. Berlin (Math. Phys.) 1915, 844 (1915).

[3] A. Einstein, Sitzungsber. Preuss. Akad. Wiss. Berlin (Math. Phys.) 1916, 688 (1916).

[4] A. Einstein, Sitzungsber. Preuss. Akad. Wiss. Berlin (Math. Phys.) 1918, 154 (1918).

[5] R. A. Hulse and J. H. Taylor, Astrophys. J. 195, L51 (1975).

[6] B. P. Abbott et al., Phys. Rev. Lett. 116, 061102 (2016).

[7] B. P. Abbott et al., Phys. Rev. X 9, 031040 (2019).

[8] R. Abbott et al., Phys. Rev. X 11, 021053 (2021).

[9] Y. Fujii and K. Maeda, The Scalar-tensor Theory of Gravitation, Cambridge Monographs on Mathematical Physics (Cambridge University Press, Cambridge, England, 2007).

[10] C. M. Will, Living Rev. Rel. 17, 4 (2014).

[11] L. Shao and N. Wex, Sci. China Phys. Mech. Astron. 59, 699501 (2016).

[12] E. Barausse, PoS KMI2017, 029 (2017).

[13] N. Wex, in Frontiers in Relativistic Celestial Mechanics: Applications and Experiments, Vol. 2, edited by S. M. Kopeikin (Walter de Gruyter GmbH, Berlin, Boston, 2014) p. 35.
[14] P. C. Freire, N. Wex, G. Esposito-Farèse, J. P. Verbiest, M. Bailes, B. A. Jacoby, M. Kramer, I. H. Stairs, J. Antoniadis, and G. H. Janssen, Mon. Not. Roy. Astron. Soc. 423, 3328 (2012).

[15] K. Yagi, D. Blas, E. Barausse, and N. Yunes, Phys. Rev. D 89, 084067 (2014), [Erratum: Phys. Rev. D 90, 069902 (2014), Erratum: Phys. Rev. D 90, 069901 (2014)].

[16] L. Shao, N. Sennett, A. Buonanno, M. Kramer, and N. Wex, Phys. Rev. X 7, 041025 (2017).

[17] J. Zhao, L. Shao, Z. Cao, and B.-Q. Ma, Phys. Rev. D 100, 064034 (2019).

[18] M. Guo, J. Zhao, and L. Shao, (2021), arXiv:2106.01622 [grqc].

[19] B. P. Abbott et al., Phys. Rev. Lett. 116, 221101 (2016), [Erratum: Phys. Rev. Lett. 121, 129902 (2018)].

[20] B. P. Abbott et al., Phys. Rev. Lett. 123, 011102 (2019).

[21] R. Abbott et al., Phys. Rev. D 103, 122002 (2021).

[22] B. P. Abbott et al., Astrophys. J. Lett. 848, L12 (2017).

[23] L. Shao, Phys. Rev. D 101, 104019 (2020).

[24] A. R. Akbarieh, S. Kazempour, and L. Shao, Phys. Rev. D 103, 123518 (2021).

[25] B. Sathyaprakash et al., Bull. Am. Astron. Soc. 51, 251 (2019). 
[26] E. Barausse, N. Yunes, and K. Chamberlain, Phys. Rev. Lett. 116, 241104 (2016).

[27] T. Damour, in Physics of Relativistic Objects in Compact Binaries: From Birth to Coalescence, edited by M. Colpi, P. Casella, V. Gorini, U. Moschella, and A. Possenti (Springer Netherlands, Dordrecht, 2009) pp. 1-41.

[28] T. Damour and G. Esposito-Farèse, Phys. Rev. D 58, 042001 (1998).

[29] B. Abbott et al., Living Rev. Rel. 23, 3 (2020).

[30] T. Akutsu et al., Nat. Astron. 3, 35 (2019).

[31] T. Akutsu et al., Prog. Theor. Exp. Phys. 2021, 05A103 (2020).

[32] D. Reitze et al., Bull. Am. Astron. Soc. 51, 035 (2019).

[33] D. Reitze et al., Bull. Am. Astron. Soc. 51, 141 (2019).

[34] S. Hild et al., Class. Quantum Grav. 28, 094013 (2011).

[35] P. Amaro-Seoane et al., (2017), arXiv:1702.00786 [astroph.IM].

[36] Z. Luo, Y. Wang, Y. Wu, W. Hu, and G. Jin, Prog. Theor. Exp. Phys 2021, 05A108 (2020).

[37] J. Mei et al., Prog. Theor. Exp. Phys 2021, 05A107 (2020).

[38] A. Sesana, Phys. Rev. Lett. 116, 231102 (2016).

[39] M. A. Sedda et al., Class. Quantum Grav. 37, 215011 (2020).

[40] M. A. Sedda et al., Exper. Astron. (2021), arXiv:2104.14583 [gr-qc].

[41] S. Sato et al., J. Phys. Conf. Ser. 840, 012010 (2017).

[42] S. Kawamura et al., (2020), arXiv:2006.13545 [gr-qc].

[43] C. Liu, L. Shao, J. Zhao, and Y. Gao, Mon. Not. Roy. Astron. Soc. 496, 182 (2020).

[44] G. Gnocchi, A. Maselli, T. Abdelsalhin, N. Giacobbo, and M. Mapelli, Phys. Rev. D 100, 064024 (2019).

[45] Z. Carson and K. Yagi, Class. Quantum Grav. 37, 02LT01 (2019).

[46] M.-S. Zhan et al., Int. J. Mod. Phys. D 29, 1940005 (2019).

[47] B. Canuel et al., Sci. Rep. 8, 14064 (2018).

[48] L. Badurina et al., JCAP 2005, 011 (2020).

[49] K. Jani and A. Loeb, (2020), arXiv:2007.08550 [gr-qc].

[50] Z. Carson, B. C. Seymour, and K. Yagi, Class. Quantum Grav. 37, 065008 (2020).

[51] E. Berti et al., Class. Quantum Grav. 32, 243001 (2015).

[52] T. Damour and G. Esposito-Farèse, Phys. Rev. Lett. 70, 2220 (1993).

[53] W. Chaibi, R. Geiger, B. Canuel, A. Bertoldi, A. Landragin, and P. Bouyer, Phys. Rev. D 93, 021101 (2016).

[54] R. X. Adhikari et al., Class. Quantum Grav. 36, 245010 (2019).

[55] M. Maggiore et al., JCAP 03, 050 (2020).

[56] I. Kowalska, T. Bulik, K. Belczynski, M. Dominik, and D. Gondek-Rosinska, Astron. Astrophys. 527, A70 (2011).
[57] C. Moore, R. Cole, and C. Berry, Class. Quantum Grav. 32, 015014 (2015).

[58] A. Freise, S. Chelkowski, S. Hild, W. Del Pozzo, A. Perreca, and A. Vecchio, Class. Quantum Grav. 26, 085012 (2009).

[59] S. Husa, S. Khan, M. Hannam, M. Pürrer, F. Ohme, X. Jiménez Forteza, and A. Bohé, Phys. Rev. D 93, 044006 (2016).

[60] S. Khan, S. Husa, M. Hannam, F. Ohme, M. Pürrer, X. Jiménez Forteza, and A. Bohé, Phys. Rev. D 93, 044007 (2016).

[61] T. M. Tauris et al., Astrophys. J. 846, 170 (2017).

[62] P. C. Peters and J. Mathews, Phys. Rev. 131, 435 (1963).

[63] J. M. Weisberg and Y. Huang, Astrophys. J. 829, 55 (2016).

[64] B. A. Jacoby, P. B. Cameron, F. A. Jenet, S. B. Anderson, R. N. Murty, and S. R. Kulkarni, Astrophys. J. Lett. 644, L113 (2006).

[65] B. Moore, M. Favata, K. G. Arun, and C. K. Mishra, Phys. Rev. D 93, 124061 (2016).

[66] N. Aghanim et al., Astron. Astrophys. 641, A6 (2020).

[67] T. Damour and G. Esposito-Farèse, Phys. Rev. D 54, 1474 (1996).

[68] S. Tahura and K. Yagi, Phys. Rev. D 98, 084042 (2018).

[69] N. Yunes and F. Pretorius, Phys. Rev. D 80, 122003 (2009).

[70] C. M. Will, Phys. Rev. D 50, 6058 (1994).

[71] M. Favata, Phys. Rev. Lett. 112, 101101 (2014).

[72] N. Loutrel, N. Yunes, and F. Pretorius, Phys. Rev. D 90, 104010 (2014), [Erratum: Phys. Rev. D 96, 089901(E) (2017)].

[73] H. Cramér, Mathematical Methods of Statistics (Princeton University Press, New Jersey, USA, 1946).

[74] C. R. Rao, in Breakthroughs in Statistics: Foundations and Basic Theory, edited by S. Kotz and N. L. Johnson (Springer, New York, USA, 1992) pp. 235-247.

[75] M. Vallisneri, Phys. Rev. D 77, 042001 (2008).

[76] C. Cutler and J. Harms, Phys. Rev. D 73, 042001 (2006).

[77] R. Schneider, V. Ferrari, S. Matarrese, and S. F. Portegies Zwart, Mon. Not. Roy. Astron. Soc. 324, 797 (2001).

[78] H.-Y. Chen, D. E. Holz, J. Miller, M. Evans, S. Vitale, and J. Creighton, Class. Quant. Grav. 38, 055010 (2021).

[79] F. Özel and P. Freire, Ann. Rev. Astron. Astrophys. 54, 401 (2016).

[80] S. E. Perkins, N. Yunes, and E. Berti, Phys. Rev. D 103, 044024 (2021).

[81] A. Toubiana, S. Marsat, S. Babak, J. Baker, and T. Dal Canton, Phys. Rev. D 102, 124037 (2020).

[82] L. S. Finn, Phys. Rev. D 46, 5236 (1992).

[83] L. S. Finn and D. F. Chernoff, Phys. Rev. D 47, 2198 (1993). 